\title{
VALORES HEMATOLÓGICOS DE LA TORTUGA MOTELO (Geochelone denticulata) MANTENIDA EN CAUTIVERIO
}

\author{
Hematological Values of the Yellow-Footed Tortoise (Geochelone \\ denticulata) RAISED IN CAPTIVITY
}
Miguel Cabrera P. ${ }^{1,2}$, Olga Li E. ${ }^{1,3}$, Hugo Gálvez C. ${ }^{4}$, Nofre Sánchez P. ${ }^{4}$, Gianmarco Rojas M. ${ }^{5}$

\section{Resumen}

\begin{abstract}
El estudio tuvo por objetivoobtener valores hematológicos de referencia de la tortuga motelo (Geochelone denticulata). Se utilizaron 44 individuos mantenidos en cautiverio en la ciudad de Iquitos-Perú. Se colectó la sangre por punción de la vena subcarapacial y se determinó recuento de glóbulos rojos (RGR), recuento de glóbulos blancos (RGB), hematocrito $(\mathrm{Ht})$, hemoglobina $(\mathrm{Hb})$, recuento diferencial de leucocitos e índices eritrocíticos. La temperatura cloacal y el peso corporal se emplearon como referencia del estado de salud. Los valores promedio fueron: RGR $0.44 \times 10^{6}$ ? L, RGB $7.82 \times 10^{3}$ ? L, Ht 20.3\%, Hb 7.0 g/dl, VCM 502.7 fl, HCM 171.4 pg, CHCM 34.1 g/dl. El recuento diferencial de leucocitos fue: Heterófilos 55.6\%, Linfocitos 25.5\%, Eosinófilos 15.8\%, Basófilos 1.5\%, Monocitos $0.4 \%$, Azurófilos $1.2 \%$. Los resultados coinciden con los intervalos de referencia documentados para la especie.
\end{abstract}

Palabras clave: Geochelone denticulata, motelo, tortuga terrestre de patas amarillas, valores hematológicos

\section{Abstract}

The objective of the study was to obtain hematological referential values for Geochelone denticulata tortoise. Blood samples of 44 tortoises, maintained in captivity in Iquitos city, Peru, were collected by puncture of the subcarapacial vein. Blood parameters under evaluation were red blood cell number (RBC), white blood cell number (WBC), hematocrit (Ht), hemoglobin ( $\mathrm{Hb})$, differential leukocyte counts and erythrocyte indexes. Cloacal temperature and body weight were used as a reference for health status.

\footnotetext{
${ }^{1}$ Laboratorio de Patología Clínica y Biología Molecular, ${ }^{4}$ Estación Experimental del Centro de Investigaciones IVITA-Iquitos, Facultad de Medicina Veterinaria, Universidad Nacional Mayor de San Marcos, Lima

${ }^{2}$ E-mail: micape17@yahoo.com

${ }^{3}$ E-mail: olgalie@hotmail.com

${ }^{5}$ Parque Zoológico Huachipa
} 
The average values were: RBC $0.44 \times 10^{6} \mathrm{~mL}, \mathrm{WBC} 7.82 \times 10^{3} \mathrm{~mL}, \mathrm{Ht} 20.3 \%$, Hb $7.0 \mathrm{~g} / \mathrm{dl}$, MCV 502.7 fl, MCH 171.4 pg, MCHC $34.1 \mathrm{~g} / \mathrm{dl}$. Leukocyte differential counts were: Heterophils 55.6\%, Lymphocytes $25.5 \%$, Eosinophils $15.8 \%$, Basophils 1.5\%, Monocytes $0.4 \%$, Azurophils $1.2 \%$. Results were similar to values reported for the species.

Key words: Geochelone denticulata, motelo, yellow-footed tortoise, hematological values

\section{INTRODUCCIÓN}

Se han descrito 17 especies de tortugas en el Perú, 15 de las cuales son de vida marina o aguas continentales y dos especies desarrollan su vida íntegramente fuera del agua. Estas últimas son la tortuga de patas rojas (Geochelone carbonaria) y la tortuga motelo (Geochelone denticulata).

En la actualidad, hay un gran interés en estudios de campo que permiten recolectar información sobre el estado de salud de poblaciones silvestres, especialmente en aquellas especies en peligro de extinción. Dentro de estos, la evaluación hematológica es un método que permite obtener excelentes indicadores del estado de salud de un individuo (Owens y Ruiz, 1980), y en el caso de los reptiles, las técnicas hematológicas han ido evolucionando favorablemente (Wilmoth, 1994; Lowell, 1998).

Existen pocos estudios sobre hematología de tortugas o testudines, entre los que se puede citar a la tortuga de caja Terrapene carolina (Herbert, 1970; Frye, 1986; Jackson, 1999; Raphael, 2003), tortuga verde Chelonia mydas y tortuga cabezona marina Caretta caretta (Herbert, 1970; Raphael, 2003), tortuga radiada Testudo radiata (Marks y Citno, 1990; Raphael, 2003), y tortuga americana Trachemys scripta elegans (Herbert, 1970; Jackson, 1999). Sin embargo, muy pocos estudios se han realizado en América Latina, entre los que destaca el estudio en la tortuga terrestre argentina Chelonoidis chilensis chilensis (Troiano y Silva, 1998).
Los valores de hemograma y bioquímica sérica obtenidos en diversas especies de testudines son muy variados, y explicables por los ecosistemas en que habita cada especie. El presente estudio tuvo por finalidad determinar los valores hematológicos de la tortuga motelo (Geochelone denticulata) como un aporte al conocimiento clínico de la especie.

\section{Materiales y Métodos}

\section{Lugar de Estudio y Animales}

El estudio se llevó a cabo en el zoocriadero comercial "Rancho Amazónico", ubicado en el distrito de San Juan Bautista, en la ciudad de Iquitos, Perú. La parte de campo se realizó en febrero de 2007, donde se tuvo temperaturas mínimas de $21^{\circ} \mathrm{C}$ y máximas de $35^{\circ} \mathrm{C}$, y una humedad relativa de $84 \%$. El procesamiento de las muestras se llevó a cabo en el Laboratorio del Centro Experimental IVITA-Iquitos de la Universidad Nacional Mayor de San Marcos (UNMSM) y en el Laboratorio de Patología Clínica de la Facultad de Medicina Veterinaria de la UNMSM.

Se emplearon 44 tortugas motelo (Geochelone denticulata), criadas en cautiverio (36 hembras y 8 machos). Las tortugas se encontraban clínicamente sanas, y eran criadas bajo las mismas condiciones de alojamiento, alimentación y control sanitario, sin distinción de sexo y edad. Se registró el peso corporal usando una balanza tipo báscula y la temperatura cloacal con un termómetro digital. 


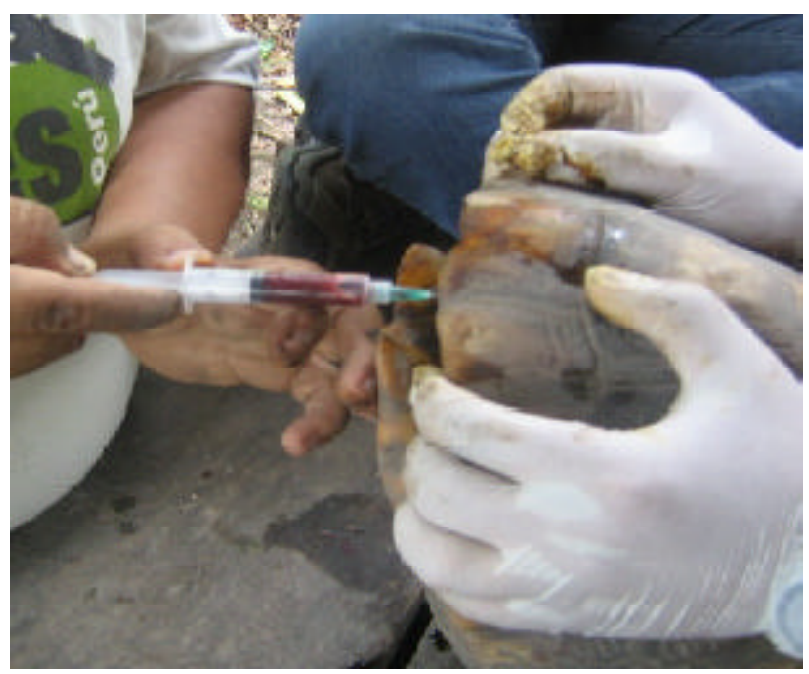

Figura 1. Toma de muestra de sangre en la tortuga motelo (Geochelone denticulata)

\section{Muestras de Sangre}

Se ensayaron varias venas para la toma de muestras (Troiano, 2004), incluyendo la vena yugular donde tuvo que usarse ketamina (22 mg/kg) para evitar el estrés causado. Finalmente, se extrajo sangre de la vena subcarapacial o de los senos cervicales dorsales (Owens y Ruiz, 1980) sin necesidad del uso de anestésico. Se introdujo una aguja 22G x 1" en ángulo perpendicular al cuello (Fig. 1), colectándose 4-5 $\mathrm{ml}$ de sangre en tubos estériles con anticoagulante de Heparina-Litio (el anticoagulante EDTA etilendiaminotetracético -, recomendado para estudios hematológicos, causa hemólisis en algunas especies de reptiles, especialmente en quelonios) (Mader, 1996).

\section{Procesamiento de Muestras}

El análisis hematológico incluyó los siguientes exámenes, según la técnica descrita por Mader (1996): Hematocrito por el método del microhematocrito; hemoglobina por el método de la cioanometahemoglobina; el recuento celular con la solución de Natt y Herrick, que permite el recuento de eritrocitos y leucocitos simultáneamente (Campbell, 1996; Molina, 2002); y el recuento diferencial de leucocitos o leucograma con la tinción Wright (Hawkey y Dennett, 1989).

Los índices eritrocíticos se obtuvieron en base a las determinaciones anteriores y aplicando las fórmulas respectivas. Se determinó el volumen corpuscular medio (VCM), la hemoglobina corpuscular media (HCM) y la concentración de hemoglobina corpuscular media (CHCM).

Para el análisis estadístico se utilizó la estadística descriptiva. Los datos se expresaron como media, desviación estándar, y valor mínimo y máximo para cada variable hematológica. Se utilizó la prueba de "t" Student para muestras independientes para determinar diferencias de la serie eritrocítica y leucocítica por efecto del sexo.

\section{Resultados y Discusión}

Factores propios del animal, como la edad, sexo, nivel de estrés, nivel nutricional, niveles hormonales e hidratación corporal, así como factores medioambientales (temperatura, estación y presión de oxígeno) afectan los valores hematológicos (Dessauer, 1970; Aguirre et al., 1995). Estas variaciones di- 
Cuadro 1. Valores promedio de la serie eritrocítica en 44 tortugas motelos mantenidas en cautiverio en un zoocriadero de la ciudad de Iquitos (2007)

\begin{tabular}{lcccc}
\hline Variables & Media & \pm & D.E. & Rango \\
\hline Eritrocitos $(\mathrm{x} \mathrm{10} / \mu \mathrm{L})$ & 0.44 & \pm & 0.19 & $0.17-0.85$ \\
Hematocrito $(\%)$ & 20.3 & \pm & 5.2 & $11-29$ \\
Hemoglobina $(\mathrm{g} / \mathrm{dl})$ & 7.0 & \pm & 2.2 & $3.1-12.2$ \\
$\mathrm{VCM}^{1}(\mathrm{fl})$ & 502.7 & \pm & 151 & $253.2-1000$ \\
$\mathrm{HCM}^{2}(\mathrm{pg})$ & 171.4 & \pm & 56.6 & $81-408$ \\
$\mathrm{CHCM}^{3}(\mathrm{~g} / \mathrm{dl})$ & 34.10 & \pm & 3.3 & $26-45.2$ \\
\hline
\end{tabular}

${ }^{1}$ Volumen corpuscular medio

${ }^{2}$ Hemoglobina corpuscular media

${ }^{3}$ Concentración de hemoglobina corpuscular media

Cuadro 2. Valores promedio de la serie leucocítica en 44 tortugas motelos mantenidas en cautiverio en un zoocriadero de la ciudad de Iquitos (2007)

\begin{tabular}{lrccc}
\hline Variables & Media & \pm & D.E. & Rango \\
\hline Leucocitos $\left(\times 10^{3} \mu \mathrm{L}\right)$ & 7.82 & \pm & 3.66 & $2.64-18.26$ \\
Heterófilos (\%) & 55.6 & \pm & 20.1 & $9-87$ \\
Linfocitos (\%) & 25.5 & \pm & 18.1 & $4-74$ \\
Eosinófilos (\%) & 15.8 & \pm & 8.9 & $3-38$ \\
Basófilos (\%) & 1.5 & \pm & 2.1 & $0-11$ \\
Monocitos (\%) & 0.4 & \pm & 0.9 & $0-4$ \\
Azurófilos (\%) & 1.2 & \pm & 2.4 & $0-12$ \\
\hline
\end{tabular}

ficultan la interpretación de los valores hematológicos observados por lo que deben tomarse en consideración en el momento de la interpretación de los análisis hematológicos (Doxey, 1987).

Los cuadros 1 y 2 muestran los valores hematológicos de las 44 tortugas motelos. El promedio de glóbulos rojos fue menor a los descritos para otros testudínidos como Geochelone carbonaria con $2.05 \times 10^{6} / ? \mathrm{~L}$ (ISIS, 1999), Chelonoidis chilensis chilensis con 0.74 x 10\%/?L, y Phrynops hilaru con $0.76 \times 10^{6} /$ ? L (Troiano, 2004). Sin embargo, los valores fueron similares a los encontrados en Testudo marginata con 0.34 x 106/?L (López-Olvera et al., 2003) y en Chelonia mydas, con $0.42 \times 10^{6} /$ ? L (Montilla et al., 2006).

El hematocrito se encontró dentro del rango de variación descrito para Phrynops hilaru (Troiano, 2004), aunque menores a los descritos para Chelonia mydas, que fue de 
29.4\% (Montilla et al., 2006) y Geochelone carbonaria (ISIS, 1999) con $29.1 \%$.

El valor de hemoglobina del presente estudio se asemeja a los reportados para Geochelone carbonaria con $7.5 \mathrm{~g} / \mathrm{dl}$ (ISIS, 1999). Sin embargo, López-Olvera et al. (2003) encontró en Testudo marginata valores de $37.3 \mathrm{~g} / \mathrm{dl}$, los cuales podrían deberse a diferencias en la alimentación (Medway et $a l, 1986)$. El valor de hemoglobina también puede variar por la técnica empleada al momento de la toma de muestra, ya que un mal manejo produciría hemólisis y, así, el escape de hemoglobina de los glóbulos rojos con aumento en sus lecturas (Benjamín, 1998).

Los índices eritrocíticos, al ser dependientes de los valores previos, mostraron un comportamiento similar al expresado por los valores hemáticos correspondientes, de allí que fueron semejantes o ligeramente diferentes a los reportados en otras especies (Troiano y Silva, 1998; ISIS, 1999; López-Olvera et al., 2003).

El número de glóbulos blancos obtenido en el presente estudio fue similar a valores reportados para algunos testudinidos como Geochelone carbonaria con $7.14 \times 10^{3} /$ ? L (ISIS, 1999), Chelonia mydas con $6.16 \times 10^{3} /$ ?L (Montilla et al., 2006) y Chelonidis chilensis chilensis con $9.81 \times 10^{3} /$ ? L(Troiano y Silva, 1998). Las diferencias entre los reportes pueden deberse a la toma de la muestra, así como a la especie, edad, actividad muscular, alojamiento y factores de estrés (Rebar et al., 2002). Por otro lado, se conoce que los valores en animales adultos son menores que en los jóvenes (Doxey, 1987).

El mayor porcentaje de células lecucocitarias correspondió a los heterófílos (55.6\%, Figs. 2 y 3). Porcentajes elevados de heterófilos han sido reportados en Chelonia mydas con $82.9 \%$ (Montilla et al., 2006). No obstante, se reportan frecuencias bastante bajas en Phrynops hilaru con $16.8 \%$ y en Chelonidis chilensis chilensis con $17.7 \%$ (Troiano, 2004). La heterofilia en reptiles está generalmente asociada a procesos inflamatorios, enfermedades bacterianas y parasitarias (Faggioni, 2006), aunque también puede ocurrir en casos de neoplasias y leucemias mieloides. Ante la aparición de enfermedades infecciosas, la heterofília puede estar acompañada de cambios en la morfología celular con la observación de heterófilos tóxicos (Rosskopf, 2000), aunque esto no ocurrió en el presente estudio. También se reporta heterofília madura en animales saludables asociada a estrés y cambios estacionales (Rosskopf, 2000). La influencia de las variaciones climáticas o ambientales sobre los valores hematológicos de reptiles es muy controversial (Rossini, 2002).

La eosinofilia se encuentra relacionada con infecciones parasitarias (Work et al., 1998). Los valores encontrados en este estudio son superiores a los observados por LópezOlvera (2003) con 8.7\%, ISIS (1999) con $6.2 \%$, y Montilla et al. (2006) con $0.5 \%$, aunque por debajo del 41.3 y $42 \%$ reportado por Troiano (2004) en Phrynops hilaru y Chelonidis chilensis chilensis.

Las monocitosis sugieren un proceso infeccioso crónico o de estimulación inmunogénica (Aguirre et al., 1995). En este estudio, los valores promedio de monocitos fueron similares a los descritos por LópezOlvera (2003). Por otro lado, la azurofilia y cambios tóxicos en la morfología celular son generalmente indicativos de infección (Mader, 1996); sin embargo, en este estudio se encontró un mínimo porcentaje de azurófilos sin cambios aparentes en la morfología celular; asimismo, los valores fueron inferiores a los descritos por Troiano (2004) en Phrynops hilaru (9.7\%) y Chelonidis chilensis chilensis $(8.7 \%)$.

No se encontró diferencia significativa por efecto del sexo en los parámetros evaluados, con excepción de los valores de heterófilos $(51.8 \pm 19.8$ vs. $72.8 \pm 10.4 \%)$ y esosinófilos (17.2 \pm 9.2 vs. $9.9 \pm 4.4 \%$ para hembras y machos, respectivamente) $(\mathrm{p}<0.05)$. 


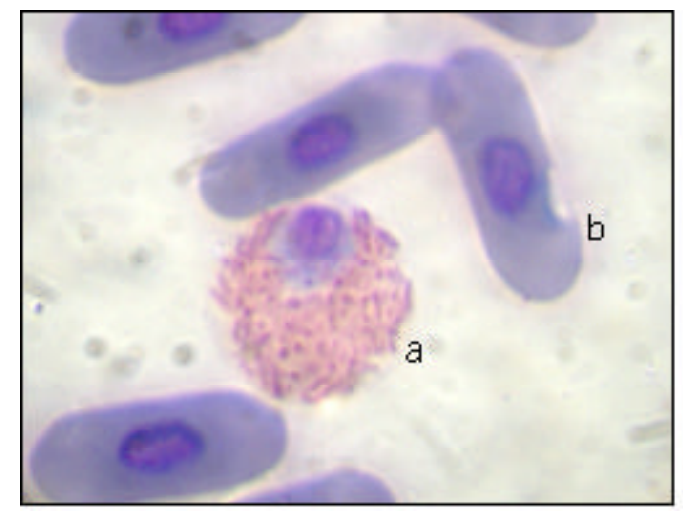

Figura 2. Heterófilo ${ }^{\mathrm{a}}$ y eritrocito ${ }^{\mathrm{b}}$ de tortuga motelo (Geochelone denticulata). Wright 100X.

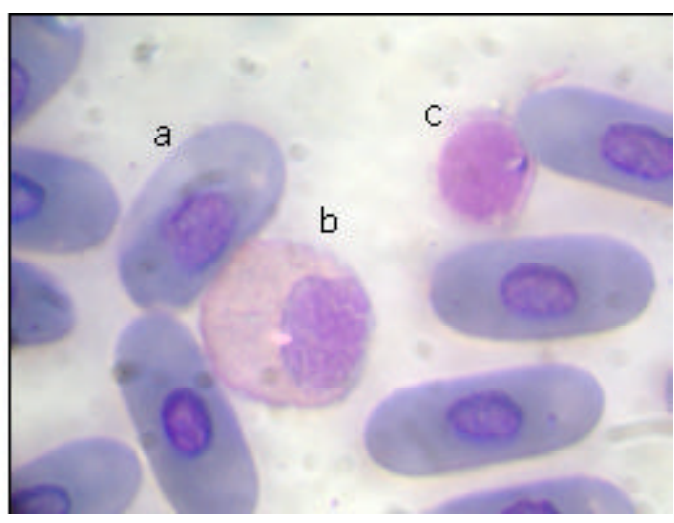

Figura 3. Eritrocito , eosinófilo $^{\mathrm{b}}$ y linfocito de tortuga motelo (Geochelone denticulata). Wright 100X.

4. Dessauer H. 1970. Blood chemistry of reptiles: Physiological and evolutionary aspects. In: Gans C, Parsons TC (eds). Biology of the reptilia, Vol 3. New York: Academic Press.1-172 p.

5. Doxey D. 1987. Patología clínica de diagnóstico en veterinaria. $2^{\mathrm{a}}$ ed. México: Ed El Manual Moderno. 316 p.

6. Faggioni C. 2006. Haemogregarines in reptiles and amphibians. University of Georgia. Athens, GA. [Internet], [14 enero 2008]. Disponible en: http:// www.vet.uga.edu/VPP/clerk/faggioni/ index.php

7. Frye F. 1986. Hematology of captive reptiles. In: Fowler ME (ed). Zoo and wild animal medicine. Philadelphia: WB Saunders. p 181-183.

8. Hawkey C, Dennett T. 1989. A color atlas of comparative veterinary hematology. USA: Iowa State University Press. 195 p.

9. Herbert C. 1970. Hematology of turtles. In: Gans C, Parsons TC (eds). Biology of the reptilia. Vol 3. p. 125-136.

10. [ISIS] International Species Information System. 1999. Valores hematológicos de la tortuga Geochelone carbonaria. [Internet], [05 septiembre 2005]. Disponible en: http:// www.isis.org.com 
11. Jackson OF. 1999. Quelonios. En: Beynon PH, Cooper JE (eds). Manual de animales exóticos. España: Sastre Vida. p 247-271.

12. Lowell A. 1998. Diagnostics procedures: hematology. En: Ackerman L (ed). The biology, husbandry and health care of reptiles. Vol III. USA: TFH Publications. p 703-713.

13. López-Olvera J, Montané J, Marco I, Martínez-Silvestre A, Soler J, Lavín S. 2003. Effect of venipuncture site on hematologic and serum biochemical parameters in marginated tortoise (Testudo marginata). J Wildlife Dis 39: 830-836.

14. Marks S, Citno Y. 1990. A field guide to western reptiles and amphibians. J Zoo Wildlife Med 21: 330-336 p.

15. Mader D. 1996. Reptile medicine and surgery. Philadelphia: WB Saunders. $512 \mathrm{p}$.

16. Medway W, Prier J, Wilkinson J. 1986. Patología clínica veterinaria. México: Ed Hispano Americana. 536 p.

17. Molina R. 2002. Hepatología y bioquímica sanguínea. En: I Encontro Ibérico de Recuperação e Conservação de Fauna Selvagem. Portugal, Lisboa: Centre de Fauna de Torreferrussa.

18. Montilla AJ, Hernández JL, Alvarado MC. 2006. Valores hematológicos de la tortuga verde (Chelonia mydas) presente en la Alta Guajira. FUC-LUZ 16: 219-226.

19. Owens D, Ruiz G. 1980. New methods of obtaining blood and cerebrospinal fluid from marine turtles. Herpetol 36: 17-20.
20. Raphael BL. 2003. Chelonias (turtles, tortoise). En: Fowler ME, Miller RE (eds). Zoo and wild animal medicine $5^{\text {th }}$ ed. Philadelphia: WB Saunders. p 48-58.

21. Rebar AP, Mac W, Metzeger F. 2002. Manual de hematología de perros y gatos. Barcelona: Multimédica. 263 p.

22. Rossini M. 2002. Determinación de los parámetros hematológicos de la Baba (Caiman crocodylus) en hábitat silvestre. Trabajo de ascenso. Maracay: Universidad Central de Venezuela. $73 \mathrm{p}$.

23. Rosskopf W. 2000. Disorders of reptilian leukocytes and erithrocytes. En: Funge A (ed). Laboratory Medicine Avian and Exotic Pets. Philadelphia: WB Saunders. p 198-204.

24. Troiano JC, Silva MC. 1998. Valores hematológicos de referencia en tortuga terrestre argentina (Chelonoidis chilensis chilensis). Analecta Veterinaria 18: 47-51.

25. Troiano JC. 2004. Valores hematológicos en tortuga terrestre argentina (Chelonoidis chilensis chilensis) y tortuga Phrynops hilaru. Hematología en Reptiles. En: Curso de hematología en Animales Silvestres. Lima: Pumas Group.

26. Wilmoth KA. 1994. Laboratory manual of reptilian hematology. 2 ed. USA: Houston Zoological Gardens. 14 p.

27. Work T, Raskin R, Balazs G, Whittaker S. 1998. Morphologic and cytochemical characteristics of blood cells from Hawaiian green turtles. Am J Vet Res 59: 1252-1257. 